Estudo da associação entre fatores socioambientais
e prevalência de parasitose intestinal
em área periférica da cidade de M anaus (AM, Brasil)

\author{
Study of the association between socio-environmental factors \\ and the prevalence of intestinal parasitosis in the suburbs \\ of the city of M anaus in the state of Amazonas, Brazil
}

Silvia Visser ${ }^{1}$

Leandro Luiz Giatti ${ }^{2}$

Ricardo Augusto Chaves de Carval ho ${ }^{1}$

JoseCamilo H urtado Guerreiro ${ }^{3}$

'Faculdadede M edicina, Escola Superior de Ciências da Saúde, U niversidade do Estado do Amazonas. Avenida Carvalho Leal

1.777, Cachoeirinha. 69065-001 M anaus AM silvisser@gmail.com ${ }^{2}$ Instituo Leônidase M aria Deane- ILM D, Fiocruz Amazônia.

${ }^{3}$ Faculdade deEnfermagem, Escola Superior de Ciências da Saúde, U niversidade do Estado do Amazonas.
Abstract This study assesses the association between socio-environmental factorsand urban sanitation conditions with the prevalence of intestinal parasitosis in a community on the periphery of the city of $M$ anaus. The study comprised a socio-environmental survey and a parasitological inquiry. A heterogeneouscommunity was revealed with some socio-economic and environmental differences between the micro-areas evaluated, even though the urban sanitation conditions were found to be predominantly precarious. The prevalance of intestinal parasitosis was $44.2 \%$. There was no significant difference between the microareas that could explain the occurrence of intestinal parasitosis. An association was found between intestinal parasitosisand residential buildingtypes, age bracket and the quality of the water used for personal hygiene and consumption in the home. Open air sewerage wasa risk factor associated with intestinal parasitosis $(O R=6.72 ; p=0.034)$ and also with intestinal protozoa ( $O R=21.87 ; p=0.004)$. In terms of the presence of protozoa, two risk factors were verified: the dumping of sewage directly into the river system $(O R=12.98 ; p=0.011)$ and the use of rudimentary cesspits $(O R=9.54 ; p=0.019)$.

Key words Intestinal Parasitosis, Health and Environment, Public Health
Resumo Este estudo teve como objetivo avaliar a associação entre fatores socioambientais e condições de saneamento urbano com a prevalência de parasitoses intestinais, em uma comunidade na periferia da cidade de M anaus. 0 estudo consistiu de um levantamento socioambiental e de um inquérito parasitológico. Foi encontrada uma comunidade heterogênea, apresentando diferenças em relação aos níveis socioeconômicos e ambientais entreasmicro-áreasavaliadas, embora as condições de saneamento básico da comunidade tenham-se mostrado precárias de uma forma geral. A prevalência de parasitoseintestinal foi de $44,2 \%$. $\mathrm{N}$ ão foi identificada diferença significativa entre as micro-áreas com respei to à ocorrência de parasitoses intestinais. H ouve associação entre parasitoses intestinais e tipo de construção residencial, faixa etária e procedência da água de higiene pessoal e do lar. 0 despejo do esgoto a céu aberto foi fator de risco associado a parasitoses intestinais $(O R=6,72 ; p=0,034)$ e a protozoários intestinais $(0 R=21,87 ; p=0,004)$. Foram fatores derisco, apenas para a presença de protozoários, o despejo de esgoto em igarapé $(O R=12,98 ; p=0,011)$ eo uso de fossa rudimentar ( $O R=9,54 ; p=0,019)$.

Palavras-chave Parasitoses intestinais, Saúde e Ambiente, Saúde Coletiva 
Introdução

\section{Saúde na Amazônia}

$\mathrm{Na}$ Amazônia, as peculiaridades ambientais exercem grande relevância para a saúde ${ }^{1}$. Esses aspectos ganham maior evidência na região de $M$ anaus, sobretudo na periferia, devido ao acelerado crescimento populacional. Becker ${ }^{2}$ assinala quanto à urbanização na Amazônia, região que teve a maior taxa de crescimento urbano do país nasúltimas décadas, onde a população vem concentrando-se sem dispor de serviços básicos adequados. É justamente nas áreas mais distantes em que há a sinergia dos fatores de risco inerentes aos processos de desmatamento para ocupação, do crescimento urbano desordenado e da precariedade de saneamento do meio. Assim, supõe-se que as populações situadas em áreas periféricas da cidade de $M$ anaus estão sujeitas a agravos associáveis tanto às paisagens naturais, como àquelas antropizadas e construídas ${ }^{1}$.

Sob a perspectiva de um melhor equacionamento de situações de insalubridade ambiental, considera-se a importância de medidas de saneamento do meio. Entretanto, quanto à implementação deinfra-estrutura e real efeito ao combate de doenças infecciosas associadas, supõe-se que exista um limiar sócio-econômico, abaixo do qual as medidas de saneamento não são suficientemente profiláticas, tendo em vista, por exemplo, que estas infecções também podem estar ocorrendo em domínio doméstico, onde a educação sanitária, as noções de higiene e os aspectos culturais apresentam relevância no controle dessas doenças ${ }^{3}$.

Coura et al. ${ }^{4}$ relacionam a presença na Amazônia degrupos populacionais com baixo padrão sócio-econômico e precárias condições sanitárias, ocasionando determinados agravos, sobretudo um alto índice de parasitoses intestinais.

\section{Parasitoses intestinais}

As enteroparasitoses ainda constituem grave problema de saúde pública para os países em desenvolvimento. Segundo Tavares-Dias et al. ${ }^{5}$, no Brasil, o problema envolvendo as parasitoses intestinais apresenta-se com uma gravidade ainda maior, em virtude da falta de políticas para uma educação sanitária profunda. Porém, sabe- seque, para a erradicação deste problema, necessita-se de melhorias nas condições socioeconômicas, no saneamento básico ena educação em saúde, além de mudanças em hábitos culturais.
Dentre as parasitoses intestinais com maior prevalência mundial encontram-se: ascaridíase, tricuríase, ancilostomíase, amebíase giardíase ${ }^{6,7}$. No Brasil, faltam dados estatísticos que mostrem a real prevalência destes microorganismos, sendo a maior parte das informações decorrentes de estudos pontuais.

Faceao exposto, o presente estudo tem como objetivo avaliar a associação entre os fatores socioambientais e as condições de saneamento urbano, com a ocorrência de parasitoses intestinais em uma comunidade, localizada em área periférica e de crescimento desordenado, na cidade de M anaus, no Estado do Amazonas.

\section{Material e métodos}

O estudo teve como alvo a população da Comunidade Vitória Régia, localizada na zona norteda cidade de M anaus, capital do Estado do Amazonas. A zona norte de $M$ anaus é caracterizada como uma área de expansão urbana recente e de constante mobilidade populacional. A comunidade encontrava-se dentro da área de cobertura do Programa de Agentes Comunitários de Saúde (PACS) e do Programa Saúde da Família (PSF), sob a abrangência da Casa de Saúde N ${ }^{\circ} .45$.

H avia 1125 famílias cadastradas nesta unidade no período derealização do estudo. Por meio deste programa, a comunidade era subdividida em 7 micro-áreas, tendo cada micro-área uma média de 160 famílias cadastradas eum Agente Comunitário deSaúde(ACS) responsável, membro da equipe do PSF. Além disso, tal comunidade possuía uma população estimada de 4271 habitantes em 2005, segundo os dados da referida Casa de Saúde.

0 estudo se baseou em um inquérito de prevalência de parasitoses intestinais, associado a uma avaliação das condições socioeconômicas, em conjunto com um diagnóstico ambiental, durante o período de janeiro a maio de 2007.

A fim de analisar as condições socioeconômicas e alguns aspectos ambientais, referentes aos domicílios da população do estudo, foram realizadas entrevistas baseadas na pesquisa mundial de saúde ${ }^{8}$, acrescidas de questões de ordem habitacional tais como: tipo demoradia, abastecimento deágua e destino de esgoto e resíduos sólidos?. Responderam à entrevista apenas os responsáveis por cada domicílio, excluindo os menores de idade. As visitas domiciliares foram acompanhadas por agentes comunitários de saúde.

O número de domicílios abordados na realização das entrevistas foi de 287. Esta amostra- 
gem considerou o total de 1125 domicílios cadastrados no PSF da comunidade Vitória Régia, o erro de 0,05 e o nível de confiança de $95 \%$. Foi escolhido o método por amostragem estratificada proporcional aleatória, considerando o número de domicílios por micro-área, incluindo os indivíduos que estiveram de acordo com a pesquisa e assinaram o Termo de Consentimento Livree Esclarecido (TCLE), após terem recebido as informações referentes aos procedimentos.

Foram realizados Exames Parasitológicos de Fezes (EPF - um examepor pessoa) em uma subamostra obtida dentre os 287 domicílios previamente determinados para entrevista. 0 tamanho da amostra para realização do EPF foi de 353 de indivíduos, dentro de uma população total de 4271, dado referente à comunidade informado pelo PSF. Seguindo o desenho da entrevista domiciliar, a amostra para a realização do EPF foi também estratificada por micro-áreas, obedecendo ao princípio da aleatoriedade.

Foram sorteados domicílios dentre aqueles que responderam à entrevista e, para cada morador dos domicílios sorteados, foi ofertada a realização do exame parasitológico defezes, atéa composição do número amostral por microárea. 0 único critério de inclusão adotado foi de idade superior a dois anos.

$\mathrm{Na}$ ocasião da entrega dos coletores para fezes, o responsável do domicílio respondia à breve entrevista sobre dados pessoais, referentes à moradia e situação clínica de cada morador. Os coletores contavam com solução de formol a 10\% e etiqueta de identificação, além de terem sido prestados esclarecimentos para a coleta do material no momento da entrega.

Os coletores com as amostras de fezes foram devolvidos pelos participantes na Casa de Saúde $\mathrm{N} \circ 45$, onde estes eram armazenados e posteriormente transportados para o laboratório realizador dos exames. Todas as amostras foram analisadas no laboratório da Policlínica Monte das Oliveiras, situado no bairro M onte das Oliveiras, pertencente à rede municipal de saúde de $M$ anaus. $A$ técnica utilizada foi o método de se dimentação espontânea ${ }^{10}$, procedimento de rotina do sistema de saúde municipal. Durante todo o período de coleta foi também realizada busca ativa domiciliar, por conta da demora na adesão dos moradores.

No total foram realizados 362 exames e, para que se alcançasse esta quantidade de amostras defezes, foi necessária a solicitação do EPF a 434 moradores, o que demonstra a mencionada dificuldade dos moradores em aderirem à pesquisa.
H ouve, assim, uma perda de 73 moradores, correspondendo a $16,8 \%$ do total entrevistado. Foi considerado como perdatodo indivíduo querespondeu ao questionário, assinou o TCLE e recebeu o coletor, porém não o devolveu com a amostra de fezes. Contudo, apesar destas perdas ocorridas, verifica-se que o número de exames de fezes realizados atendeu ao estipulado inicialmente pelo plano amostral do estudo.

Através da observação direta da área foi efetuado um diagnóstico ambiental, com caráter descritivo, de situações ambientais que pudessem constituir fatores de risco para a ocorrência de parasitoses intestinais, tais como: disposição inadequada de resíduos sólidos, presença de esgoto a céu aberto e presença de lixo doméstico no peridomicílio. Este diagnóstico ambiental foi registrado por meio de fotografias digitais.

Os resultados das entrevistas e dos exames de fezes foram armazenados em planilhas do programa M icrosoft Excel ${ }^{\circledR}$, eposteriormenteforam utilizados os programas EPI IN FO ® , versão 2005, de distribuição gratuita pelo CDC/Atlanta, e M initab Statistical Software ${ }^{\circledR}$, versão 14.2.

$\mathrm{N}$ a estatística descritiva, utilizou-seo número absoluto e a frequência relativa das variáveis de maior relevância para a construção de tabelas. Estas variáveis foram: sexo, idade, grau deinstrução, tipo de construção de residência, tipo de piso da residência, renda per capita, número de pessoas por domicílio, procedência da água para beber e cozinhar, procedência da água para higiene pessoal e do lar, tratamento da água para beber e cozinhar, destino do esgoto, destino do lixo e, por fim, presença de parasitos intestinais no EPF.

A associação entreas variáveisfoi analisada por meio da regressão logística binária, considerando como variável dependente os resultados dos exames parasitológicose, como variáveisindependentes, os fatores sócio-econômicos eambientais. Foi utilizado o teste de $\chi^{2}$ para analisar a existência de associação entre as variáveis ${ }^{11}$, e considerado o nível de significância inferior a $0,05(p \leq 0,05)$.

0 presente estudo atendeu às diretrizes enormas da Resolução no 196/96 do Conselho N acional de Saúde, e foi aprovado pelo Comitê de Ética em Pesquisa da Fundação de M edicina Tropical do Amazonas (FM T-AM) em 18 de outubro de 2006.

\section{Resultados}

Foram entrevistados os responsáveis de 296 domicílios, abrangendo 1351 moradores, e foram realizados 362 exames defezes, atendendo ao pla- 
no amostral inicialmente estipulado do estudo. A distribuição da amostragem nas micro-áreas é apresentada na Tabela 1.

Dos moradores entrevistados, 673 (49,8\%) eram do sexo masculino e $678(50,2 \%)$ do sexo feminino. A média deidadefoi de 22,7 anos $\pm 17,5$.

Sobre o nível educacional dos moradores, a maioria, $640(56,8 \%)$, possuía ensino fundamental incompleto, $41(3,6 \%)$ eram analfabetos, 94 $(8,4 \%)$ eram alfabetizados, $61(5,4 \%)$ possuíam ensino fundamental completo, $84(7,5 \%)$ ensino médio incompleto, 179 (15,9\%) ensino médio completo, $16(1,4 \%)$ ensino superior incompleto eape nas $11(1,0 \%)$ possuíam ensino superior comple to. Dos entrevistados, $278(20,6 \%)$ eram crianças em faixa etária não escolar e, do total, 225 moradores não informaram o grau de instrução.

Dentre os respondentes das entrevistas domiciliares, 131 (44,3\%) possuíam ensino fundamental incompleto, $14(4,7 \%)$ eram analfabetos,
$22(7,4 \%)$ eram alfabetizados, $24(8,1 \%)$ possuíam ensino fundamental completo, 21 (7,1\%) ensino médio incompleto, $74(25,0 \%)$ ensino mé dio completo, seis $(2,0 \%)$ ensino superior incompleto e apenas quatro $(1,4 \%)$ possuíam ensino superior completo.

A média de renda per capita mensal encontrada foi de $R \$ 186,78$, com desvio padrão (DP) de 300,3 ( $n=1351$ ), sendo o salário mínimo da época de $R \$ 380,00$. A média de habitantes por cômodo foi de 1,8 $(n=294)$. E a maioria das casas visitadas (51,7\%) possuía de 4 a 6 residentes.

$\mathrm{Na}$ Tabela 2 encontra-se a média de renda per capita mensal, a distribuição de residências segundo o tipo de construção e a procedência da água para beber e cozinhar, segundo a micro-área.

Dentreostipos depiso nos domicílios, a grande maioria, 257 (87,4\%), informou possuir piso de alvenaria. 0 chão batido foi encontrado em $21(7,1 \%)$ domicílios, a madeira em $11(3,7 \%)$ e

Tabela 1. Distribuição da amostragem segundo divisão de micro-área.

\begin{tabular}{lcccc}
\hline Micro-área & $\begin{array}{c}\mathrm{N}^{\circ} \text { dedomicílios } \\
\text { amostrados }\end{array}$ & $\begin{array}{c}\mathrm{N}^{\circ} \text { de moradores } \\
\text { entrevistados }\end{array}$ & $\begin{array}{c}\mathrm{N}^{\circ} \text { de entrevistados } \\
\text { (EPF) }\end{array}$ & $\begin{array}{c}\mathrm{N}^{\circ} \text { de exames } \\
\text { parasitológicos realizados }\end{array}$ \\
\hline M A 1 & 41 & 183 & 72 & 59 \\
M A 2 & 35 & 166 & 53 & 43 \\
M A 3 & 45 & 204 & 64 & 55 \\
M A 4 & 48 & 230 & 73 & 59 \\
M A 5 & 47 & 222 & 73 & 62 \\
M A 6 & 46 & 197 & 54 & 53 \\
M A 7 & 34 & 149 & 46 & 31 \\
Total & 296 & 1351 & 435 & 362 \\
\hline
\end{tabular}

EPF: Exame parasitológico de fezes.

Tabela 2. Distribuição da média de renda per-capita, água para beber e cozinhar e tipo de construções de casas de acordo com as micro-áreas.

\begin{tabular}{|c|c|c|c|c|c|c|c|c|c|c|c|c|c|c|c|}
\hline \multirow{3}{*}{$\begin{array}{l}\text { Micro- } \\
\text { área }\end{array}$} & \multirow{3}{*}{$\begin{array}{l}\text { M édia de renda per } \\
\text { capita mensal (n) }\end{array}$} & \multicolumn{8}{|c|}{ Procedência da água para beber e cozinhar* } & \multicolumn{6}{|c|}{ Tipo de construção** } \\
\hline & & \multicolumn{2}{|c|}{$\begin{array}{c}\text { Água } \\
\text { encanada }\end{array}$} & \multicolumn{2}{|c|}{$\begin{array}{c}\text { Água } \\
\text { mineral }\end{array}$} & \multicolumn{2}{|c|}{$\begin{array}{c}\text { Poço } \\
\text { cacimba }\end{array}$} & \multicolumn{2}{|c|}{$\begin{array}{l}\text { Poço } \\
\text { profundo }\end{array}$} & \multicolumn{2}{|c|}{ Alvenaria } & \multicolumn{2}{|c|}{$\begin{array}{l}\text { Madeira e } \\
\text { alvenaria }\end{array}$} & \multicolumn{2}{|c|}{ Madeira } \\
\hline & & $\mathrm{n}$ & $\%$ & $\mathrm{n}$ & $\%$ & $\mathrm{n}$ & $\%$ & $\mathrm{n}$ & $\%$ & $\mathrm{n}$ & $\%$ & $\mathrm{n}$ & $\%$ & $n$ & $\%$ \\
\hline MA 1 & $\mathrm{R} \$ 221,9$ & 0 & 0 & 3 & 7,3 & 31 & 75, & 7 & 17, & 30 & 75,0 & & 10, & 6 & 15,0 \\
\hline MA 2 & $\mathrm{R} \$ 190,00 \pm 188,00(166)$ & 0 & 0 & 0 & 0 & 30 & 85,7 & 5 & 14,3 & 25 & 71,4 & 8 & 22,9 & 2 & 5,7 \\
\hline MA 3 & $R \$ 239,60 \pm 550,90(204)$ & 0 & 0 & 3 & 6,7 & 29 & 64,4 & 13 & 28,9 & 31 & 68,9 & & & 9 & 20,0 \\
\hline MA 4 & & 0 & 0 & 1 & 2,1 & 43 & & 4 & & 31 & 64,6 & 7 & & 10 & 20,8 \\
\hline MA 5 & $\mathrm{R} \$ 139,76 \pm 91,95(217)$ & 11 & 23,4 & 0 & 0 & 24 & 51,1 & 12 & 25,5 & 34 & 72,3 & 4 & 8,5 & 9 & 19,2 \\
\hline MA 6 & $R \$ 118,60 \pm 176,00(202)$ & 11 & 23,9 & 1 & 2,2 & 34 & 73,9 & 0 & 0 & 21 & 45,7 & 4 & 8,7 & 21 & 45,6 \\
\hline MA 7 & $R \$ 285,60 \pm 407,50(149)$ & 30 & 88,2 & 0 & 0 & 4 & 11,8 & 0 & 0 & 23 & 67,6 & 2 & 5,9 & 9 & 26,5 \\
\hline Total & $R \$ 186,78 \pm 300,3(1351)$ & 52 & 17,6 & 8 & 2,7 & 195 & 65,9 & 41 & 13,9 & 195 & 66,1 & 34 & 11,5 & 66 & 22,4 \\
\hline
\end{tabular}

*10 domicílios não forneceram informação; ${ }^{* *} 1$ domicílio não forneceu informação. 
o piso misto de madeira e alvenaria foi referido em quatro ( $1,4 \%)$. A penas um $(0,3 \%)$ domicílio possuía piso de alvenaria e chão batido e dois $(0,7 \%)$ não forneceram informação.

Quando avaliado o fornecimento de água, foi encontrada uma comunidade de uma forma geral desprovida de abastecimento, eque utilizava principal mente água de poço de lençol freático (cacimba) para beber e cozinhar (Tabela 2).

Em relação ao tratamento realizado na água para beber e cozinhar, 146 (49,8\%) domicílios informaram não realizar nenhum tratamento, $113(38,7 \%)$ acrescentavam hipoclorito, e 34 $(11,5 \%)$ utilizavam métodos diversos de tratamento da água, sendo a fervura referida em 11 (3,7\%) domicílios e a filtragem em 21 (7,1\%) domicílios. 0 método de decantação foi citado em apenas um $(0,3 \%)$ domićlíio e a associação de fervura e hipoclorito também foi citado em um $(0,3 \%)$ domicílio. Três ( $1,0 \%)$ domicílios não forneceram informação. Dentre os domicílios que utilizavam o poço tipo cacimba como fonte de água para beber e cozinhar, 87 (44,6\%) não realizavam qualquer tipo de tratamento.

A água utilizada pelos moradores para uso de higiene pessoal edo lar foi referida em 241 (81,4\%) domicílios de proveniência de poço tipo cacimba, em 41 (13,9\%) domicílios de água encanada, 12 $(4,1 \%)$ de poço tipo profundo e dois $(0,7 \%)$ domicílios não responderam a esta pergunta.

Quando questionado sobre o destino do esgoto, a grande maioria dos domicílios utilizava a fossa rudimentar, sendo esta situação declarada em 190 (64,2\%) domicílios do total entrevistado. Chama a atenção o número de domicílios que despejavam seu esgoto a céu aberto, $22(7,4 \%)$, bem como diretamente no igarapé, $65(22,0 \%)$, sem nenhuma forma de tratamento. Apenas 14 $(4,7 \%)$ dos domicílios referiram possuir rede pública de esgoto.

Em relação ao destino final do lixo doméstico, $258(87,2 \%)$ domicílios informaram utilizar a coleta pública municipal. Queimavam o seu lixo $14(4,7 \%)$ domicílios e $9(3,0 \%)$ despejavam 0 lixo no entorno. Uma minoria, cinco $(1,7 \%)$ dos domicílios, relatou mais deum destino para o seu lixo, utilizando a coleta pública, o despejo no entorno eno igarapé ea queima. N ove (3,0\%) relataram outros destinos para o lixo doméstico.

Apesar da relativa boa cobertura por serviço público de coleta deresíduos sólidos, foi possível observar acúmulos de resíduos domésticos a céu aberto em diversas áreas peridomiciliares, inclusive em locais com bom acesso por ruas pavimentadas e com atendimento por coleta pública.
No inquérito parasitológico, dentre os 362 moradores que realizaram o exame de fezes, 205 $(56,6 \%)$ eram do sexo feminino e, 157 (43,4\%) do sexo masculino. $E$, em relação à idade, a distribuição por faixa etária pode ser verificada na Tabela 3.

A prevalência de parasitoses intestinais encontrada neste estudo foi de $44,2 \%$, dos quais $55,0 \%$ eram monoparasitados e $45,0 \%$ poliparasitados. A prevalência para helmintosfoi de $11,0 \%$ e de $37,3 \%$ para protozoários. Houve infecção mista para helmintos e protozoários em 10,6\% dos exames.

Em relação à faixa etária, encontrou-se a seguinte distribuição de resultados: 1a) entre 2 e 6 anos, 37 exames positivose 45 negativos; 2a) de 7 a 14 anos, 47 positivos e 36 negativos; 3a) de 15 a 17 anos, nenhum exame positivo e sete negativos; 4a) de 18 a 60 anos, 69 positivos e 100 negativos; e5ㄹ) maiores de 60 anos, sete positivose 14 negativos.

Foram pesquisadas 13 espécies de parasitose comensais intestinais, e entre os helmintos a maior prevalência encontrada foi deAscarislumbricoides com $28(70,0 \%)$ exames positivos, seguido pelo H ymenolepis nana com nove $(22,5 \%)$; Trichuris trichiura com sete (17,5\%); Ancylostoma duodenalis com três (7,5\%); Enterobius vermiculares com um (2,5\%); e não foi encontrado exame positivo para Strongyloides stercoralis.

Entre os protozoários, a maior prevalência foi paraEntamoeba coli, com 57 (42,2\%) exames positivos, seguida por Endolimax nana com 53 (39,3\%); Giardia lamblia e E. hystolitica/díspar ambos com $37(27,4 \%)$ positivos; lodamoeba butschlii com $11(8,1 \%)$ eBalantidium coli eBlastocystis hominis com um $(0,7 \%)$ exame positivo para cada.

$\mathrm{Na}$ totalidade de exames realizados, encontrou-se a seguinte prevalência de comensais in-

Tabela 3. Distribuição dos exames de fezes conforme a faixa etária.

\begin{tabular}{lrc}
\hline \multicolumn{1}{c}{ Faixa etária } & $\begin{array}{c}\mathrm{N}^{\circ} \text { de } \\
\text { participantes relativa (\%) }\end{array}$ & $\begin{array}{c}\text { Frequência } \\
\text { 2 }\end{array}$ \\
\hline a 6 anos (pré-escolar) & 82 & 22,7 \\
7 a 14 anos (escolar) & 83 & 22,9 \\
15 a 17 anos (adolescente) & 7 & 1,9 \\
18 a 60 anos (adultos) & 169 & 46,7 \\
M ais de 60 anos (idosos) & 21 & 5,8 \\
Total & 362 & 100,0
\end{tabular}


testinais: E. coli de $15,7 \%$, E. nana de $14,6 \%$ e, I. butschlii de 3,0\%.

Em relação à divisão das micro-áreas da comunidade, obteve-se a seguinte distribuição de resultados positivos para algum parasito intestinal, helmintos e protozoários (Tabela 4).

Dentreosindivíduos que realizaram o exame de fezes, $227(62,7 \%)$ residiam em casa de alve naria, $90(24,9 \%)$ residiam em casa de madeira e $45(12,4 \%)$ em casa de alvenaria e madeira.

A maioria dos participantes, 249 (68,8\%), afirmou queutilizava água proveniente de poço tipo cacimba para beber e cozinhar, $58(16,0 \%)$ utilizava o poço tipo profundo, $53(14,6 \%)$ a água encanada e dois $(0,6 \%)$ a água mineral.

Quando avaliados em conjunto a procedência da água utilizada para higiene elar eo resultado do exame de fezes, verificou-se que a mai oria dos participantes, $315(87,0 \%)$, respondeu ser usuária da água proveniente de poço tipo cacimba e, entre estes, $146(46,3 \%)$ tiveram seus exames positivos para al gum parasito intestinal.

Em relação à faixa etária, observou-se que crianças de idade escolar apresentaram maior prevalência de parasitoses intestinais do queadolescentes eadultos. Essa diferença, de acordo com a faixa etária, foi estatisticamentesignificante $\left(\chi^{2}=\right.$ $12,55 ; p=0,01369)$. Porém, a análise de tendência linear não mostrou diferenças significativas $\left(\chi^{2}=\right.$ 2,$995 ; p=0,0835$ ).

Quando analisado o tipo de construção, observou-se uma maior prevalência de parasitos intestinais em moradores de residência de madeira $\left(\chi^{2}=10,17 ; p=0,00619\right)$.

Em relação à associação entre a prevalência deparasitos intestinais com a procedência daágua para higiene e do lar, encontrou-se um número maior deresultados positivos nos indivíduos que utilizavam água proveniente de poço cacimba $\left(\chi^{2}=7,6 ; p=0,02288\right)$.

$\mathrm{N}$ a regressão logística binária, o despejo do esgoto a céu aberto $(O R=6,72 ; p=0,034)$ se mostrou um fator de risco para parasitoses intestinais. 0 despejo do esgoto em igarapé $(O R=12,98$; $p=0,011)$, em fossa rudimentar ( $O R=9,54$; $p=0,019)$ ea céu aberto $(O R=21,87 ; p=0,004)$ se mostraram fatores de risco para a infecção por protozoários (Tabela 5).

A construção da residência de alvenaria-madeira foi fator de proteção para parasitoses intestinais ( $O R=0,31 ; p=0,005)$, helmintoses $(O R=0,14 ; p=0,032)$ e protozooses $(O R=0,31$; $p=0,005$ ).

\section{Discussão}

A prevalência das parasitoses intestinais encontrada nestetrabal ho foi um pouco inferior quando comparada a outros estudos realizados na cidade de $M$ anaus, como aquele de $M$ artins et al. ${ }^{12}$, com uma prevalência de $58,4 \%$ no bairro Parque das $\mathrm{N}$ ações, área também atendida pelo PSF. Chagas et al..$^{13}$ encontraram uma prevalência de $89,5 \%$ em uma comunidade rural nas proximidades da cidade de $M$ anaus.

Outras pesquisas também demonstraram alta prevalência das parasitoses intestinais, como as realizadas por $\mathrm{M}$ artins et al. ${ }^{14}$, que encontraram $74,3 \%$ na comunidade de N ossa Senhora de Fátima, e por Tavares et al. ${ }^{15}$, que acharam uma prevalência de $87 \%$ no assentamento TarumãM irim.

Predomina nestes estudos a indicação das condições do saneamento básico, principalmente aquelas referentes à coleta e ao armazenamen-

Tabela 4. Distribuição de resultados de exames de fezes de acordo com a micro-área.

\begin{tabular}{|c|c|c|c|c|c|c|c|}
\hline \multirow[t]{2}{*}{ Micro-área } & \multirow{2}{*}{$\begin{array}{l}\text { Exames } \\
\text { realizados } \\
n\end{array}$} & \multicolumn{2}{|c|}{$\begin{array}{l}\text { Exames } \\
\text { positivos }\end{array}$} & \multicolumn{2}{|c|}{ Helmintos } & \multicolumn{2}{|c|}{ Protozoários } \\
\hline & & $\mathrm{n}$ & $\%$ & $\mathrm{n}$ & $\%$ & $\mathrm{n}$ & $\%$ \\
\hline MA 1 & 59 & 29 & 49,2 & 9 & 15,3 & 25 & 42,4 \\
\hline MA 2 & 43 & 15 & 34,9 & 4 & 9,3 & 12 & 27,9 \\
\hline MA 3 & 55 & 25 & 45,5 & 6 & 10,9 & 21 & 38,2 \\
\hline MA 4 & 59 & 25 & 42,4 & 5 & 8,5 & 24 & 40,7 \\
\hline MA 5 & 62 & 27 & 43,5 & 6 & 9,7 & 22 & 35,5 \\
\hline MA 6 & 53 & 27 & 50,9 & 7 & 13,2 & 22 & 41,5 \\
\hline MA 7 & 31 & 12 & 38,7 & 3 & 9,7 & 9 & 29,0 \\
\hline Total & 362 & 160 & 44,2 & 40 & 11,0 & 135 & 37,3 \\
\hline
\end{tabular}


Tabela 5. Regressão logística entre variáveis sócio-ambientais e parasitoses, helmintoses e protozooses.

\begin{tabular}{llrl}
\hline \multicolumn{1}{c}{ Exposição } & \multicolumn{1}{c}{ Categorias } & OR (IC a 95\%) & P \\
\hline Fatores associados a parasitoses intestinais & & & \\
$\quad$ Tipo de construção* & Alvenaria-madeira & $0,31(0,14-0,70)$ & 0,005 \\
$\begin{array}{l}\text { Disposição do esgoto** } \\
\text { Fatores associados a helmintoses }\end{array}$ & Céu aberto & $6,72(1,15-39,25)$ & 0,034 \\
$\quad$ Tipo de construção* & Alvenaria-madeira & $0,14(0,02-0,85)$ & 0,032 \\
Fatores associados a protozooses & & & \\
$\quad$ Tipo de construção* & Alvenaria-madeira & $0,38(0,17-0,88)$ & 0,024 \\
Disposição do esgoto** & Igarapé & $12,98(1,82-92,56)$ & 0,011 \\
& Fossa rudimentar & $9,54(1,46-62,33)$ & 0,019 \\
& Céu aberto & $21,87(2,68-178,61)$ & 0,004 \\
\hline
\end{tabular}

${ }^{*}$ categoria de comparação = alvenaria; ${ }^{* *}$ categoria de comparação = rede coletora.

to da água utilizada para consumo, ao destino do lixo caseiro e a hábitos de higiene, como fatores que não só favorecem a elevada prevalência das parasitoses intestinais, mas também contribuem para a manutenção destas.

Quando avaliamos a infecção por al guns parasitos intestinais transmitidos por penetração de larvas, como o ancilostomídeos e Strongyloides stercoralis, foi encontrada uma baixa prevalência no presente estudo. No caso do S. stercoralis nenhuma amostra foi positiva para este helminto. Este fato pode ser justificado pelo método utilizado, já que a sedimentação espontânea não éa metodologia adequada para o diagnóstico desta espécie. Para tal finalidade, o método de escolha é o de pesquisa de larvas, ou método Baermann- M oraes e de Rugai ${ }^{16}$.

Podemos afirmar que a metodologia empregada para o estudo também não foi apropriada para o diagnóstico de E. vermiculares, visto a baixa prevalência encontrada deste helminto. 0 melhor método para encontrar ovos deste parasito é o de Graham (método da fita gomada) ${ }^{17}$. Contudo, o método de sedimentação foi o escoIhido para este estudo, por ser o método utilizado na rede pública de saúde. Em virtude destas situações, podemos julgar que muitas infecções podem não estar sendo diagnosticadas devido à técnica da rede de saúde pública.

Neste estudo encontrou-se uma população com baixo grau de escolaridade, onde a maioria dos moradores não possuía o ensino fundamental completo (56,8\%). Entre os participantes do inquérito parasitológico, foi encontrada diferença relativa no nível educacional destes quando avaliados conforme a micro-área. A pesar disso, não houve associação entreo nível educacional e a ocorrência de parasitoses intestinais, nem diferença de prevalência de parasitoses intestinais entre as micro-áreas. Todavia, é importante salientar queo nível educacional, enquanto uma característica geral da população, é um fator importante para a compreensão das doenças, das formas de transmissão e de sua prevenção.

Somado à baixa escolaridade, outro fator importante para a compreensão, a transmissão e, principal mente, a prevenção das doenças, vem a ser o papel exercido pel os Agentes Comunitários de Saúde junto à comunidade, por meio de promoção da saúde ${ }^{18}$, e também através de mudanças nas concepções das populações acerca de suas necessidades de saúde e de melhorias em seu bem-estar ${ }^{19}$.

Quanto à prevenção de infecções por parasitos intestinais, foi observado nessa comunidade que quase a metade dos domicílios questionados não utilizava nenhum método de tratamento da água consumida. $E$, dentre os domicílios que utilizavam água do poço, cacimba para beber e cozinhar, quase a metade também não realizava nenhum tratamento da água.

0 resultado encontrado nesta pesquisa não condiz com a prática da educação continuada em saúde, proposta pela estratégia Saúde da Família do PSF. Este fato demonstra, em parte, o descumprimento do objetivo da promoção da saúde, podendo este ser decorrente de uma precária capacitação do agente comunitário para a função exercida, ou por outros aspectos associados à não inserção do mesmo na comunidade, seja por inadequação profissional, por fator cultural de uma não aceitação de sua presença pela própria comunidadeou, ainda, pelo fato do agente não residir na área de trabalho. 
No que diz respeito à ação destes agentes, foi observada neste trabalho uma variação entre as micro-áreas em relação à adesão dos participantes ao inquérito parasitológico. Na micro-área 6, quase a totalidade dos entrevistados realizaram o exame de fezes, havendo apenas um (1,9\%) desistente. Já na micro-área 7, para atingir a meta amostral de 31 exames, foram necessárias 46 entrevistas, o que revelou um índice de 33,6\% de desistência.

Uma provável explicação para esta variação pode ter sido a representatividade de cada agente comunitário em sua micro-área de atuação, visto que as visitas foram realizadas com a presença destes. Portanto, podemos, com estes dados, ressaltar a importância da inserção do agente desaúde na comunidade para o efetivo exercício de sua função na educação e na promoção da saúde.

Em relação à avaliação do nível social e econômico, foi encontrada uma comunidade debaixa renda familiar, onde a renda per capita mensal era menor do que um salário mínimo em mais de $90 \%$ dos moradores. A composição das residências era em grande parte formada por um núcleo familiar constituído por um grupo de 4 a 6 pessoas, e a média de habitantes por cômodo foi de 1,8. Destemodo, verifica-se, de uma forma geral, uma comunidade constituída por um congestionamento domiciliar, no qual os espaços domésticos podem ser de uso múltiplo e, assim, facilitadores de doenças que possuam transmissão interpessoal.

Neste contexto, ao se analisar o estudo realizado por Souza et al. ${ }^{20}$, em um assentamento agrícola em Granada no Estado do Acre, verifica-se que a média de habitantes por cômodo encontrada foi de 1,1, e a prevalência de parasitose intestinal foi de $53,4 \%$. Fortes et al. ${ }^{21}$ demonstraram em seu estudo que indivíduos residentes em domicílios que possuíam mais de um morador por cômodo, além de renda familiar abaixo de um salário mínimo, apresentaram maior chance de ocorrência de A. lumbricoides. Pedrazzani et al..$^{22}$ analisaram a prevalência de helmintos intestinais e sua associação com renda e tamanho da família, anemia e estado nutricional em escolares em São Paulo. Destas associações, houve resultado estatisticamente significante apenas para 0 tamanho familiar. Vale ressaltar que o salário mínimo per capita é um índice sócio-econômico no qual o tamanho familiar essencialmente figura em sua conceituação.

Em relação à condição de moradia, observou-se que a maioria das residências era construída em alvenaria, e 33,9\% das casas era de madeira ou de construção mista de madeira e alvenaria. Cerca de $87,0 \%$ das casas possuíam piso de alvenaria; 0 chão batido esteve presente em $7,1 \%$, emadeira em $3,7 \%$ do total. Na análise de associação entre parasi toses intestinais e piso das residências, não foi encontrada diferençasignificativa em relação aos tipos de piso. Contudo, diferença estatisticamentesignificantefoi encontrada entre parasitoses intestinais e residências construídas em madeira, assim como o estudo de Basualdo et al. ${ }^{23}$, realizado numa área rural da Argentina, o qual demonstrou a associação entre a presença de parasitos e algumas variáveis ambientais, entre as quais: casas de madeira, piso dechão batido, bomba d'água comunitária efossa rudimentar.

Sabe-se que o conceito de saneamento básico compreende 0 abastecimento de água e a disposição de esgotos, a coleta e a disposição de lixo, a drenagem urbana e o controle de vetores ${ }^{24}$. No presente estudo foram avaliados a procedência da água, o destino de esgoto e do lixo esua associação com a ocorrência de parasitoses intestinais.

A comunidade apresentava um sistema precário de rede pública coletora de esgoto, onde a maioria das casas utilizava a fossa rudimentar como destino final deseu esgoto (64,2\%). A penas uma minoria $(4,7 \%)$ dispunha de rede pública de esgoto, e o despejo a céu aberto, ou diretamente em igarapés sem nenhuma forma de tratamento, foi reportado em $29,4 \%$ dos domicílios.

0 uso de água sem tratamento, contaminada por dejetos humanos, é considerado uma forma frequente de contaminação por alguns parasitos intestinais, como por exemplo pela E. hystolytica ${ }^{25}$ e a G. lamblia ${ }^{26}$. Além disso, é um fator determinante para 0 alto índice de parasitos de veiculação hídrica. A via de contaminação ocorre não só através da ingestão de água contaminada, mas também através do banho, na higiene pessoal ou por alimentos contaminados.

Este estudo demonstrou uma prevalência para E. hystolytica $(23,1 \%)$ compatível com outros estudos realizados em áreas semel hantes na cidade de $M$ anaus, como o de Martins et al. ${ }^{14}$, que encontrou uma prevalência de $29,5 \%$ deste protozoário. Entretanto, avaliando a prevalência de G. Iamblia $(23,1 \%)$, encontrou-se uma maior comparada com o resultado de $M$ artins et al. ${ }^{27}$, que foi de $14,1 \%$. Outros estudos também mostraram prevalência menor de G. Iamblia, como o de Coura et al. ${ }^{4}$, com 5,7\%, Rios et al. ${ }^{28}$, com $8,6 \%$ e Souza et al. ${ }^{20}$, com $0,5 \%$. É importante salientar que o resultado encontrado poderia ser maior, uma vez que o ideal seria a execu- 
ção de três exames de fezes com intervalo de sete dias entre cada um ${ }^{26}$.

Um dado que pode ser usado como indicador de contaminação fecal da água consumida é a infecção por comensais intestinais. N este estudo, a prevalência da infecção pela Entamoeba coli epela Endolimax nana foi de $35,6 \%$ e $33,1 \%$, respectivamente. Esteresultado podeindicar a contaminação da água utilizada por dejetos humanos. E, por observação direta, constatou-se que alguns domicílios tinham sua fossa rudimentar próxima ao poço cacimba, o que poderia ser fonte de vazamento e contaminação da água.

A infecção por alguns geohelmintos, por exemplo, pelo A. lumbricoides, também ocorre através do uso de água sem tratamento, contaminada com ovos ${ }^{29}$. Porém, esta ocorre primordialmente através da contaminação do ambiente com ovos de helmintos no solo ou no piso das habitações. As precárias condições ambientais decorrentes da insalubridade das habitações são descritas como fatores favoráveis para o aumento das infecções por helmintos.

No que se refere à procedência da água, foi encontrada uma comunidade com críticas condições de abastecimento, onde apenas $17,4 \%$ dos domicílios utilizavam água proveniente do sistema público para beber e cozinhar, e 65,9\% utilizavam água de poço cacimba para este consumo. Quando questionada a procedência da água para higiene pessoal e do lar, o percentual de domicílios que utilizavam o poço cacimba aumentou para $81,4 \%$, e os que utilizavam água encanada do sistema público diminuíram para 13,9\%.

Avaliando a procedência da água por microárea, foi visto que quatro delas (micro-área 1, 2, 3 e4) eram completamente desprovidas deabastecimento público, fazendo a grande maioria uso da água do poço cacimba. Já a micro-área 7 possuía satisfatória rededeabastecimento público deágua, onde $88,2 \%$ das residências utilizavam esta fonte de água para beber e cozinhar, e 85,2\% também a utilizavam para higienepessoal e do lar. A pesar dos resultados demonstrarem diferenças entre as micro-áreas quanto à origem da água, tanto para beber e cozinhar, quanto para higiene pessoal e do lar, não houve diferença no estudo quanto à prevalência de parasitoses entre estas. Contudo, foi encontrada diferença significativa na ocorrência de parasitoses intestinais quando avaliada a procedência da água para higiene pessoal e do lar. Este fato demonstra a existência, neste estudo, de uma associação entre a ocorrência de parasitoses intestinais e a utilização de água proveniente de poço cacimba para uso na higiene pessoal e do lar.
Alguns fatores ambientais foram estudadose associados a helmintos intestinais em crianças por Teixeira et al. ${ }^{30}$, sendo evidenciado que o fator de maior risco para estas infecções está associado à disposição dos esgotos no terreno ou nas ruas $(\mathrm{OR}=3,474 ; p=0,002)$. No presente estudo foi demonstrado um maior risco para parasitoses intestinais quando associado à disposição do esgoto a céu aberto $(O R=6,72 ; p=0,034)$. E houvetambém evidências do risco el evado para protozoários intestinais quando o esgoto doméstico teve como destino final o igarapé $(O R=12,98 ; p=0,011)$, a fossa rudimentar $(O R=9,54 ; p=0,019)$ e o despejo a céu aberto $(O R=21,87 ; p=0,004)$. Estes dados evidenciam quea falta de saneamento constitui risco para parasitoses intestinais.

Por meio do diagnóstico ambiental, foi constatada a presença de grande quantidade de lixo em áreas peridomiciliares, porém $87,2 \%$ dos entrevistados responderam utilizar o serviço municipal de coleta delixo para a destinação final de seus resíduos. Uma vez havendo tal serviço de coleta de lixo à disposição dos moradores, a dispersão de dejetos ao redor dos domicílios também éum elemento que indica inadequadas práticas sanitárias, contribuindo para um ambiente peridomiciliar não saudável, e também para a proliferação de vetores que contribuem na disseminação de doenças infecciosas, tais como as parasitoses intestinais.

0 estudo de Rios et al..$^{28}$ analisou a qualidade da água de abastecimento na população indígena de lauaretê, no município de São Gabriel da Cachoeira, no Estado do Amazonas, e realizou exames parasitológicos na população e em amostras de solo. Das amostras de água analisadas, $89,2 \%$ estavam contaminadas com coliformes termotolerantes. A prevalência de A . lumbricoides em exames de fezes foi de $64,8 \%$ e, em amostras de solo contaminadas, foi de $52,6 \%$ para ovos deste parasito, demonstrando a contaminação do solo e da água por contaminantes fecais, bem como a exposição da população aos riscos de infecção.

\section{Consideraçõesfinais/Conclusões}

Diante dos resultados, constatamos claramente a existência de uma comunidade exposta a riscos de infecção por enteroparasitos, principalmente em decorrência da falta de saneamento básico.

Como prováveis principais causas de infecção, verificamos a ocorrência de contato direto da população com solo contaminado por resíduos sólidos e fezes, bem como a utilização de 
água proveniente de fontes de captação próximas a lençol freático possivelmente contaminado. A presença de residências construídas com paredes e pisos de madeira, assim como piso de chão batido, propicia o acúmulo de poeira e a possível contaminação do ambiente por ovos de geohelmintos.

No que se refere aos aspectos sócio-econômicos, esperava-se encontrar uma maior prevalência de parasitoses intestinais em comparação com outros estudos, assim como também era esperada diferença nas prevalências entre as micro-áreas, considerando os distintos aspectos de renda, moradia e abastecimento de água. Entretanto, esta diferença não foi encontrada no presente estudo, fato que pode ser decorrente de outros aspectos comportamentais e ambientais não explorados nesta pesquisa, como por exemplo, o hábito de lavagem de mãos, o uso prévio de medicação anti-helmíntica eanti-protozoária e a presença de animais e insetos no domicílio.

Concordando com Possas ${ }^{31}$, que considera a complexidade de fatores de exposição e de tolerância de populações humanas a doenças infecciosas, somada às limitações das ciências socioeconômicas e ecológicas, há uma necessidade de serem estabelecidas alternativas de estudos sob a perspectiva transdisciplinar de saúde no ecossistema social. Além desta complexidade, ressalta- mos a importância de uma equipe de saúde de família ativa e representativa, que exerça a função de educação na concepção ena promoção de saúde e bem- estar.

Diante da realidade estudada, e considerando que as parasitoses intestinais são relativamente bem compreendidas em relação aos seus mecanismos de transmissão, enten de-se que o controle das mesmas vai além das questões meramente sanitárias, demandando ações no campo referentes às condições de vida. Além disso, faz-senecessária uma mudança do perfil de trabalho em saúde, visando o efetivo envolvimento da comunidade em torno da promoção da saúde e da melhoria da qualidadedevida, culminando em um processo de desenvolvimento sócio-cultural ${ }^{19}$.

De fato, abordagens participativas e intersetoriais vêm sendo frequentemente aplicadas na implementação do PACS e do PSF ${ }^{19}$. Contudo, políticas de saúde pública, bem como necessidades de implantação de infraestrutura de saneamento básico, fazem-se prementes para o enfrentamento das precárias condições socioambientais da comunidade apresentadas e analisadas neste trabal ho. Além disso, estas ações devem ser complementadas através do desenvolvimento de uma equipe de saúde de família ativa e devidamente capacitada, a qual, de fato, exerça sua função de promoção da saúde e bem-estar da população.

\section{Colaboradores}

S Visser e LL Giatti trabal haram no delineamento da pesquisa, coleta de dados, elaboração do artigo e revisão do texto; RAC Carvalho participou na revisão crítica do texto; JCH Guerreiro trabalhou na interpretação de dados, análise estatística e revisão crítica do texto.

\section{Agradecimentos}

A Eduardo Guimarães M erçon, por seu apoio na revisão do texto. 


\section{Referências}

1. Confalonieri UEC. Saúde na Amazônia: um modelo conceitual para análise de paisagens e doenças. Estudos Avançados 2005; 19(53):221-36.

2. Becker BK. Geopolítica da Amazônia. Estudos Avançado. 2005; 19(53):71-86.

3. Soares SRA, Bernardes RS, Cordeiro-N etto OM. Relações entre saneamento, saúde pública e meio ambiente: elementos para formulação de um modelo de planejamento em saneamento. Cad Saude Publica 2002; 18(6):1713-24.

4. Coura JR, Wilcox HPF, Tavares AM, Paiva DD, Fernandes $\mathrm{O}$, Rada ELJC, Eusébio P, Perez EP, Borges LCL, H idalgo MEC, Nogueira M LC. Aspectos epidemiológicos, sociais e sanitários de uma área no Rio Negro, Estado do Amazonas, com especial referência às parasitoses e à infecção chagásica. Cad Saude Publica 1994; 10(Supl. 2):327-336.

5. Tavares-Dias M, Grandini AA. Prevalência e aspectos epidemiológicos de enteroparasitoses na população de São José da Bela Vista, São Paulo. Revista da Sociedade Brasileira de Medicina Tropical 1999; 32(1):63-65

6. Mandel G, Bennett J, Dolin R. Principles and practice of infectious diseases. 4a.ed. v. 2. N ew York: Churchil Livingstone; 1995.

7. Castiñeira TM PP, Martins FSV. Infecções por helmintos e enteroprotozoários. Centro de Informação em Saúde para Viajantes - CIVES. Rio de Janeiro: UFRJ. 2002. [acessado em 2006 jun 30]; Disponível em: http://www.cives.ufrj.br/informes/helmintos/ hel-0y.pdf

8. Szwarcwald CL, Viacava F, Vasconcellos MTL, Leal MC, Azevedo LO, Queiroz RSB, Carvalho MF, Fonseca M G, Frias PF, Gama SG, Souza Júnior PRB, Lansky S, Luhm KR, M azzei MC, Ribeiro M CSA, Santos S, Souza WV, Theme M. O Brasil em números. Radis 2004; 23:14-33.

9. Gioia I. Levantamento eco-parasitário da população residente na Fazenda Intervales - SP. [tese]. São Paulo (SP): Faculdade de Saúde Pública de São Paulo; 1995.

10. Neves DP. Exame parasitológico de fezes. In: Parasitologia Humana. 8a ed. São Paulo: Ed. Atheneu; 1991. p. 461-471.

11. Vieira S. Introdução à bioestatística. $3^{3}$. ed. Rio de Janeiro: Editora Campus; 1980.

12. $M$ artins $M$, Soares $A R L$, M oura MAS, Borborema CAB. Perfil Parasitológico no Bairro Parque das nações, $M$ anaus - AM, Atendidos Pelo Programa M édico da Família. In: XXXVII Congresso da Sociedade Brasileira de M edicina Tropical, 2001, Salvador/BA. Revista da Sociedade Brasileira de M edicina Tropical 2001; 34:309.

13. Chagas ECSC. Relação entre parasitismo intestinal ea variabilidade dos níveis de hemoglobina, eosinófilos, proteínas totais e estado nutricional na comunidade rural Nossa Senhora do Livramento, M anaus - AM. [dissertação]. M anaus(AM ): Universidade do Estado do Amazonas; 2004.
14. Martins $M$, Alecrim W, Tavares AM, Soares ARL, M oura M AS, Chagas EC. Parasitoses intestinais na comunidade Nossa Senhora de Fátima, M anaus AM. In: XXXIX Congresso da SBMT, 2003, Belém/ PA. Revista da Sociedade Brasileira de M edicina Tropical 2003; 36:184.

15. Tavares AM, Abtibol MRA, Ferreira CR, Assis TMA, Veloso IC, Martins M, Soares ARL. Enteroparasitoses em moradores do assentamento do Incra no Tarumã-M irim, M anaus - AM . In: XLII Congresso da Sociedade Brasileira de M edicina Tropical, 2006, Teresina/PI. Revista da Sociedade Brasileira de M edicina Tropical 2006; 39:206.

16. Costa-Cruz JM. Strongyloides stercoralis. In: Parasitologia Humana. $11^{a}$ ed. São Paulo: Ed. Atheneu; 2005. p. 275-284.

17. Neves DP. Enterobius vermicularis. In: Parasitologia Humana. 11 a ed. São Paulo: Ed. Atheneu; 2005. p. 285-288.

18. Silva JA, Dalmaso ASW. O agente comunitário de saúde e suas atribuições: os desafios para os processos de formação de recursos humanos em saúde. Interface - Comunicação, Saúde, Educação 2002; 6(10):75-96.

19. Teixeira CF. Promoção e vigilância da saúde no contexto da regionalização da assistência à saúde no SUS. Cad Saude Publica 2002; 18(Supl.):153-162.

20. Souza EA, Silva-Nunes $M, M$ alafronte RS, M uniz PT, Cardoso MA, Ferreira MU. Prevalence and spatial distribution of parasitic infections in a rural Amazonian settlement, Acre State, Brazil. Cad Saude publica 2007; 23(2):427-434.

21. Fortes BPM D, Valencia LIO, Ribeiro SV, Medronho RA. Modelagem geoestatística da infecção por Ascaris lumbricoides. Cad Saude Publica 2004; 20(3): 727-734.

22. Pedrazzani ES, M ello DA, Pripas S, Fucci M, Barbosa CAA, Santoro M CM. H elmintoses Intestinais. II - Prevalência e correlação com renda, tamanho da família, anemia e estado nutricional. Rev Saude publica 1988; 22(5):384-389.

23. Basualdo JA, Córdoba MA, De Luca M M, Ciarmela ML, Pezzani BC, Grenovero MS, M invielle MC. Intestinal parasitoses and environmental factors in a rural population of Argentina, 2002-2003. Rev. Inst. M ed. trop. S.Paulo 2007; 49(4):251-255.

24. Soares SRA, Bernardes RS, Cordeiro-N etto OM. Relações entre saneamento, saúde pública e meio ambiente: elementos para formulação de um modelo de planejamento em saneamento. Cad Saude Publica 2002; 18(6):1713-1724.

25. Silva EF, Gomes MA. Amebíase: Entamoeba histoIytica/Entamoeba díspar. In: Parasitologia Humana. 11ạ ed. São Paulo: Ed. Atheneu; 2005. p. 127138.

26. Sogayar MITL, Guimarães S. Giardia. In: Parasitologia Humana. 11a ed. São Paulo: Ed. Atheneu; 2005. p. $121-126$ 
27. Martins M, Tavares AM, M oura MAS, Soares ARL, Soares AS, Celestino E, Ribeiro IAC. Parasitoses intestinais em pacientes atendidos pelo Programa Saúde da Família em um bairro de M anaus-AM. In: XXXIX Congresso da SBMT, 2003, Belém/PA. Revista da Sociedade Brasileira de M edicina Tropical 2003; 36:185.

28. Rios L, Cutolo SA, Giatti LL, Castro M, Rocha AA, Toledo RF, Pelicioni M CF, Barreira LP, Santos JG. Prevalência de parasitos intestinais e aspectos socioambientais em comunidade indígena no Distrito de Iauaretê, Município de São Gabriel da Cachoeira (AM ), Brasil. Saúde Soc. São Paulo 2007; 16(2):76-86.

29. Silva AVM, Massara CL. Ascaris lumbricoides. In: Parasitologia Humana. 11a ed. São Paulo: Ed. Atheneu; 2005. p. 253-259.

30. Teixeira JC, Heller L. Fatores ambientais associados às helmintoses intestinais em área de assentamento subnormal, Juiz de Fora, MG. Eng. sanit. ambient. 2004; 9(4):301-305.

31. Possas CA. Social ecosystem health: Confronting the complexity and emergence of infectious diseases. Cad Saude Publica 2001; 17(1):31-41.

Artigo apresentado em 01/07/2008

Aprovado em 10/02/2009

Versão final apresentada em 27/02/2009 